\title{
Dopamine, Corticostriatal Connectivity, and Intertemporal Choice
}

\author{
Andrew S. Kayser, ${ }^{1,2,3}$ Daicia C. Allen, ${ }^{1,2}$ Ana Navarro-Cebrian, ${ }^{1,2}$ Jennifer M. Mitchell, ${ }^{1,2}$ and Howard L. Fields ${ }^{1,2}$ \\ ${ }^{1}$ Department of Neurology, University of California, San Francisco, San Francisco, California 94143, ${ }^{2}$ Ernest Gallo Clinic and Research Center, Emeryville, \\ California 94608, and ${ }^{3}$ Department of Neurology, Veterans Affairs Northern California Health Care System, Martinez, California 94553
}

Value-based decisions optimize behavioral outcomes. Because delayed rewards are discounted, an increased tendency to choose smaller, immediate rewards can lead to suboptimal choice. Steep discounting of delayed rewards (impulsivity) characterizes subjects with frontal lobe damage and behavioral disorders including substance abuse. Correspondingly, animal studies and indirect evidence in humans suggest that lower dopamine in the frontal cortex contributes to steeper discounting by impairing corticostriatal function. To test this hypothesis directly, we performed a randomized, double-blind, counterbalanced, placebo-controlled study in which we administered the brain penetrant catechol-O-methyltransferase inhibitor tolcapone or placebo to healthy subjects performing a delay discounting task. Tolcapone significantly increased choice of delayed monetary rewards, and this tolcapone-induced increase covaried with increased BOLD activity in the left ventral putamen and anterior insula. Tolcapone also changed corticostriatal connectivity: specifically, by inducing a decrease in the coherence between ventral putamen and pregenual cingulate cortex. These results indicate that raising cortical dopamine levels attenuates impulsive choice by changing corticostriatal function.

\section{Introduction}

Intertemporal comparison and selection of different available rewards is critical to optimal decision making (Carter et al., 2010; Peters and Büchel, 2011). A tendency to choose immediate over delayed rewards even when the immediate reward is significantly smaller (delay discounting) can be quantified by measuring the ratio of smaller, sooner monetary rewards chosen by a subject when a larger, later reward is available. Steeper discounting of delayed rewards is associated with frontal brain injury (Sellitto et al., 2010) and with multiple substance use disorders, including abuse of alcohol, opiates, and psychostimulants (Kirby et al., 1999; Petry, 2001; Coffey et al., 2003). For example, we previously showed that a delay discounting measure, the impulsive choice ratio (ICR), correlates significantly with performance on the widely used and validated Barratt Impulsivity Scale, and that abstinent alcoholics show significantly steeper discounting of delayed rewards (Mitchell et al., 2005; Boettiger et al., 2009).

Received March 9, 2012; revised May 14, 2012; accepted May 17, 2012.

Author contributions: A.S.K., J.M.M., and H.L.F. designed research; A.S.K. and D.C.A. performed research; A.S.K. contributed unpublished reagents/analytic tools; A.S.K. and A.N.-C. analyzed data; A.S.K., J.M.M., and H.L.F. wrote the paper.

This work was supported by funding from the Wheeler Center for the Neurobiology of Addiction, Telemedicine and Advanced Technology Research Center Grants W81XWH-10-1-0231 and W81XWH-11-1-0596 (J.M.M., A.S.K., H.L.F.), Alcoholic Beverage Medical Research Foundation/The Foundation for Alcohol Research (A.S.K.), and funds from the State of California (H.L.F., A.S.K.). We thank Dawn Weinstein and Candice Teague for essential help with subject screening, and the subjects themselves for their participation.

The authors declare no competing financial interests.

Correspondence should be addressed to Andrew S. Kayser, Department of Neurology, University of California, San Francisco, Ernest Gallo Clinic and Research Center, 5858 Horton Street, Suite 200, Emeryville, CA 94608. E-mail: akayser@gallo.ucsf.edu.

DOI:10.1523/JNEUROSCI.1180-12.2012

Copyright $\odot 2012$ the authors $\quad 0270-6474 / 12 / 329402-08 \$ 15.00 / 0$
Recent studies indicate that levels of dopamine modulate impulsivity (Dagher and Robbins, 2009; Volkow et al., 2009), and delay discounting behavior in particular. For example, a high (20 $\mathrm{mg}$ ) but not low (10 mg) dose of D-amphetamine decreased delay discounting in healthy controls when choices were based on the amount but not the probability of a later reward (de Wit et al., 2002). Conversely, Pine et al. (2010) demonstrated that relative to placebo, control subjects given the dopamine precursor L-dopa showed consistent increases in delay discounting. In addition, the genotype for the dopamine-degrading enzyme catechol-Omethyltransferase (COMT) correlates with levels of impulsivity: subjects tend to be more impulsive if they have alleles encoding the more enzymatically active form of COMT (Boettiger et al., 2007). Because COMT represents the primary route for dopamine degradation in frontal cortex (Gogos et al., 1998), lower dopamine tone in frontal cortex may therefore predispose some individuals to greater impulsivity.

Reconciling the apparent paradox that either increases or decreases in dopamine can result in greater impulsivity may thus require closer consideration of the brain locus of alteration in dopamine levels. In most brain regions, dopamine is inactivated by reuptake into nerve terminals through the dopamine transporter. However, the density of dopamine transporter is low in prefrontal cortex and consequently COMT has a significant influence on cortical synaptic dopamine levels (Chen et al., 2004). In fact, mice with inactivated COMT genes show increased cortical but not subcortical (striatal) dopamine levels (Gogos et al., 1998). The possibility therefore arises that discounting of delayed rewards depends on the balance between cortical and striatal dopamine tone (Cools, 2008). In particular, whereas increases in striatal dopamine may increase impulsive choice, selectively increasing dopamine tone in frontal cortex - via COMT inhibition, 
or possibly via inhibition of cortical dopamine transporter activity at higher drug concentrations-may reduce discounting of delayed rewards. In this study, we hypothesized that a single 200 $\mathrm{mg}$ dose of the brain penetrant COMT inhibitor tolcapone, by preferentially increasing dopamine tone in frontal cortex, would reduce impulsive choice and increase BOLD activity in brain regions associated with selection of larger, later rewards.

\section{Materials and Methods}

Subject training and task performance. We screened 45 subjects, of whom 27 healthy subjects (i.e., without a history of neurological or psychiatric illnesses) were eligible to participate. All subjects gave written informed consent in accordance with the Committee for the Protection of Human Subjects at the University of California, San Francisco, and University of California, Berkeley. Subjects first underwent a history and physical exam, as well as blood testing for liver function, to ensure that there were no medical contraindications to tolcapone use or MRI scanning. They then completed a number of screening questionnaires, including the Barratt Impulsivity Scale (BIS). One subject was excluded before behavioral analysis for use of an albuterol inhaler immediately before MRI scanning. Three subsequent subjects were excluded after task performance: one reported an explicit change in discounting strategy before the second session, one was noted to be significantly sleep-deprived after completing final exams the day of the second session, and a third was a non-native English speaker who may have misunderstood task instructions. The differential ICR (see below) of the first of these excluded subjects favored tolcapone $(-0.59)$ and of the other two favored placebo $(0.45,0.92)$; all values were identified as significant outliers $(p<0.005)$ by Rosner's generalized extreme studentized deviate (ESD) procedure (Rosner, 1983). Of the 23 subjects who remained, ages ranged from 19 to 41 years; 13 were female. All subjects had normal neuroanatomy as reviewed by a neurologist (A.S.K.), were right-handed, and had normal or corrected-to-normal vision. Before scan sessions, subjects were trained on the delay discounting task to familiarize them with task procedures. Subjects then underwent two $2.25 \mathrm{~h}$ fMRI sessions consisting of 8 runs of 32 task trials, for a total of 256 trials, and two resting state runs. All subjects were compensated for their participation.

Experimental paradigm. Subjects were randomized in double-blind, counterbalanced, placebo-controlled fashion to either placebo or a single $200 \mathrm{mg}$ dose of tolcapone on their first visit and the alternative treatment on their second visit. After receiving task instructions and undergoing a brief practice session of 10-20 trials, subjects performed the delay discounting task (see Fig. 1) within the MRI scanner while blood-oxygen level dependent (BOLD) images were obtained. Subjects entered the MRI scanner $90 \mathrm{~min}$ after drug ingestion to ensure that the delay discounting task was performed while drug levels were at peak $(\sim 120 \mathrm{~min}$ : tolcapone package insert, Valeant Pharmaceuticals). The 256 total trials were presented in pseudorandom order. At the start of each trial, subjects were cued to one of four trial types: Want (W), Don't Want (DW), Sooner (S), and Larger (L) (see below). For each of these trial types, subjects were then presented with two hypothetical alternatives: a smaller amount of money available today ("Now") and a larger amount available later ("Later"). We have previously shown that this paradigm with hypothetical rewards effectively engages subjects (Boettiger et al., 2007, 2009), consistent with reports that hypothetical rewards activate common brain regions involved in value computations (Kang et al., 2011). As in our previous work (Boettiger et al., 2007), the Later option consisted of six amounts $(\$ 1, \$ 2, \$ 5, \$ 10, \$ 20$, or $\$ 100)$ at one of five future delays (1 week, 2 weeks, 1 month, 3 months, or 6 months). The percentage difference between the Now and Later options was selected from one of four different values $(30,15,10$, and $5 \%)$. Subjects then made a button press to select one of the two options, randomly assigned to the left and right sides of the screen.

Each of the four trial types allowed us to investigate different functions. In the $\mathrm{W}$ condition, the primary analytic focus of this study, subjects chose the option they preferred. In the DW condition, subjects also chose the option they preferred, but then made a button press to select the opposite choice. This condition permitted us to evaluate motor im- pulsivity by comparing the proportion of responses in the W and DW conditions. In the $\mathrm{S}$ and $\mathrm{L}$ conditions, which we combined to form a control (CON) condition, subjects simply selected the sooner or larger options, respectively. These trial types allowed us to ensure that subjects were appropriately following instructions, and to introduce a condition in which the decision about monetary options was not a motivated choice. The W condition comprised one-half of all trials; the CON conditions comprised one-third; and the DW condition comprised onesixth. As expected, subjects performed very well in the CON condition [accuracy, $0.97 \pm 0.008(\mathrm{SEM})$ ], and therefore we do not further report results of the CON condition in this paper.

The primary behavioral outcome was the ICR, which represents the ratio of the number of sooner choices to the number of total choices in the $\mathrm{W}$ condition. As in our previous work, we calculated a number of related measures of impulsive choice, including a measure of the hyperbolic discounting rate $(k)$ and the interest rate differentiating the two choices. Because values such as the hyperbolic discounting rate were highly correlated with ICR (e.g., $r(\mathrm{ICR}, k)=0.78 ; p<1 \times 10^{-5}$ ) and consequently gave qualitatively similar results, we elected to study the simplest, most intuitive quantity, as in our previous work (Boettiger et al., 2007, 2009). ICR values underwent an arcsine-square root transform (i.e., were variance-stabilized) to permit the application of parametric statistical tests.

Both before drug administration and after the scanner run, subjects completed a speeded responding task to assess potential changes in motor function on and off tolcapone. Subjects were required to make a button press response as soon as possible after the presentation of either a brief visual or auditory stimulus. Reaction times were compared both within each session and across the tolcapone and placebo conditions. Due to computer failures, these data were available for only 19 of our 23 subjects.

MRI image acquisition. MRI scanning was conducted on a Siemens MAGNETOM Trio 3T MR Scanner at the Henry H. Wheeler, Jr., Brain Imaging Center at the University of California, Berkeley. Anatomical images consisted of 160 slices acquired using a T1-weighted MP-RAGE protocol (TR, $2300 \mathrm{~ms}$; TE, $2.98 \mathrm{~ms}$; FOV, $256 \mathrm{~mm}$; matrix size, $256 \times$ 256; voxel size, $1 \mathrm{~mm}^{3}$ ). Functional images consisted of 24 slices acquired with a gradient echoplanar imaging protocol (TR, $1370 \mathrm{~ms}$; TE, $27 \mathrm{~ms}$; FOV, $225 \mathrm{~mm}$; matrix size, $96 \times 96$; voxel size, $2.3 \times 2.3 \times 3.5 \mathrm{~mm}$ ). A projector (Avotec SV-6011; http://www.avotec.org) was used to display the image on a translucent screen placed within the scanner bore behind the head coil. A mirror was used to allow the subject to see the display. Subjects made their responses via an MRI-safe fiber optic response pad (Inline Model HH-1x4-L; http://www.crsltd.com).

fMRI preprocessing. fMRI preprocessing was performed using both the AFNI (http://afni.nimh.nih.gov) and FSL (http://www.fmrib.ox.ac.uk/ fsl/) software packages. Functional images were converted to 4D NIfTI format and corrected for slice-timing offsets. Motion correction was performed using the AFNI program 3 dvolreg, with the reference volume set to the mean image of the first run in the series. Images were then smoothed with a $5 \mathrm{~mm}$ FWHM Gaussian kernel. Coregistration was performed with the AFNI program 3dAllineate using the local Pearson correlation cost function optimized for fMRI-to-MRI structural alignment. The subsequent inverse transformation was used to warp the anatomical image to the functional image space. Anatomical images were normalized to a standard volume (MNI_N27) using the FSL program fnirt available from the Montreal Neurological Institute (MNI) (http:// www.bic.mni.mcgill.ca). The same normalization parameters were later applied to native-space statistical maps as necessary for the generation of group statistical maps (see below).

Univariate analysis. To address a series of hypotheses, we performed a number of voxelwise fMRI statistical analyses for each subject using the general linear model (GLM) framework implemented in the AFNI program 3dDeconvolve. The BOLD correlates of different decisions were assessed by modeling the cue and decision phases of the task for the four different conditions (Want, Don't Want, Sooner, Larger) with separate regressors, each of which was derived by convolving a gamma probability density function (peaking at $6 \mathrm{~s}$ ) with a vector of stimulus onsets for each condition. Mapwise significance ( $p<0.05$, corrected for multiple com- 
parisons) was determined by applying a cluster-size correction derived from the AFNI programs $3 d F W H M x$ and $3 d$ ClustSim on data initially thresholded at a value of $p<0.005$ (uncorrected). For the main effect of task (see Fig. 3), a whole-brain correction was applied. This contrast compared event onsets from all conditions-including both cue and outcome-with baseline fMRI activity on tolcapone versus placebo to illustrate that there are widespread changes in BOLD signal across the brain resulting from the use of tolcapone. Because cortical dopamine projections are frontally predominant (Cools, 2008), subsequent univariate analyses designed to address more specific task-related hypotheses about changes in frontostriatal regions used the AAL template brain (Tzourio-Mazoyer et al., 2002) to generate a frontostriatal mask (AAL areas 3-32 and 71-76; analyses illustrated in Figs. 4 and $5 a$ ).

Multivariate analysis. Resting state data were used to evaluate the connectivity between brain regions in both the tolcapone and placebo conditions in all subjects who completed both resting state sessions ( $N=22$; due to technical failures, complete resting state data from 1 of our 23 subjects were not obtained). This approach allowed us to identify any drug-related changes in connectivity independent of task performance. These data were smoothed by a $2.5 \mathrm{~mm}$ FWHM Gaussian kernel before temporal bandpass filtering between 0.009 and $0.08 \mathrm{~Hz}$ to reduce the influence of cardiac and respiratory artifact, per the protocol of Fox et al. (2005). Movement parameters and the white matter and ventricular time series, but not the global mean signal, were included as regressors of no interest. A striatal region of interest in the left ventral putamen was chosen, based on the results of our univariate analyses (see Fig. 4a). A time course defined by averaging across voxels in this region was then correlated with every other voxel in the brain, and correlation coefficients were Fisher-transformed to allow for the application of parametric statistical tests. Images were then normalized to the MNI template before the application of group-level statistics. Because of our hypotheses about changes in frontostriatal connectivity, data were masked with a prefrontal mask (AAL regions 3-32) before the appropriate cluster size correction was calculated.

For the evaluation of connectivity during task performance, we used the seed region (left ventral putamen) and significantly connected region (left pregenual anterior cingulate cortex) identified in the resting state connectivity analysis shown in Figure $5 b$. For each of these two ROIs, the time series for each of the eight task-state runs was windowed with a 4-point split-cosine bell and concatenated with the other segments to produce a subject-specific time series. Time series within each ROI were then averaged across voxels to generate a single time series for each ROI. Coherency values were obtained by applying a fast Fourier transform (MATLAB; http://www.mathworks. com) to the data for each pair of ROIs, implemented via Welch's periodogram averaging method using a 64-point discrete Fourier transform, Hanning window, and overlap of 32 points (Kayser et al., 2009). Coherence values for each ROI were then computed using the band-averaged coherence. To compute correlations between coherence results and other values, we first Fisher-transformed the coherence values to generate an approxi- a

Figure 1. Task structure. $\boldsymbol{a}$, Each trial of the delay discounting task began with fixation, followed by a cue to the trial type. After iittered delay, subjects were prompted to make a decision (in this case, a "Want" decision). $\boldsymbol{b}$, Illustrated are the four trial types: Want, Don't Want, Sooner, and Larger (see Materials and Methods). The latter two trial types comprised the control ("Con")

a
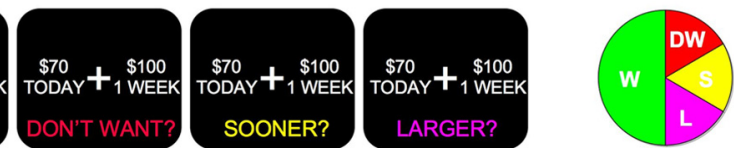

3.5-7.5s

Time

b
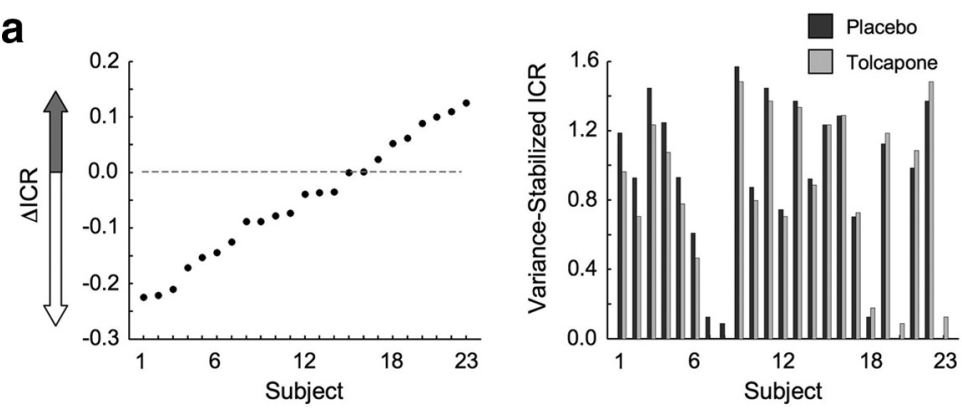

b

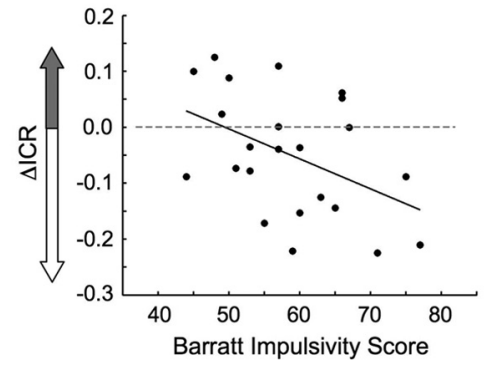

Figure 2. Behavioral results. $\boldsymbol{a}$, Shown are the differences between the variance-stabilized impulsive choice ratios $[\Delta I C R=I C R$ (tolcapone) - ICR (placebo)], ordered by magnitude across the 23 subjects (left), as well as the actual ICR values for both placebo and tolcapone (right). There was a significant decline in ICR across the group in the tolcapone condition: $\Delta I C R=-0.04, p=$ 0.025. $\boldsymbol{b}$, The effect of tolcapone varied with an independent measure of baseline impulsivity $(r=-0.45 ; p=0.032)$. Specifically, $\triangle I C R$ was negatively correlated with the score on the BIS, where higher BIS scores indicate greater impulsivity.

mately normal distribution (Rosenberg et al., 1989) that permitted us to apply parametric statistical tests.

Statistical analysis. For analysis of behavioral data, paired $t$ tests and Pearson's correlation coefficients were used to calculate statistical significance. For univariate and multivariate analyses of BOLD data, significance was calculated using statistical techniques and corrections implemented in the Afni software package, including the functions 3dDeconvolve, 3dFWHMx, 3dClustSim, and 3dttest ++ .

\section{Results}

Twenty-three subjects completed a delay discounting task (Fig. 1) on both days of the study and were eligible for analysis. Subjects exhibited a range of ICRs (the number of sooner choices divided by the total number of choices in the Want condition) that spanned 0 (no sooner choices) to 1 (all sooner choices). Across the group, a significant reduction in ICR was seen in the tolca- 

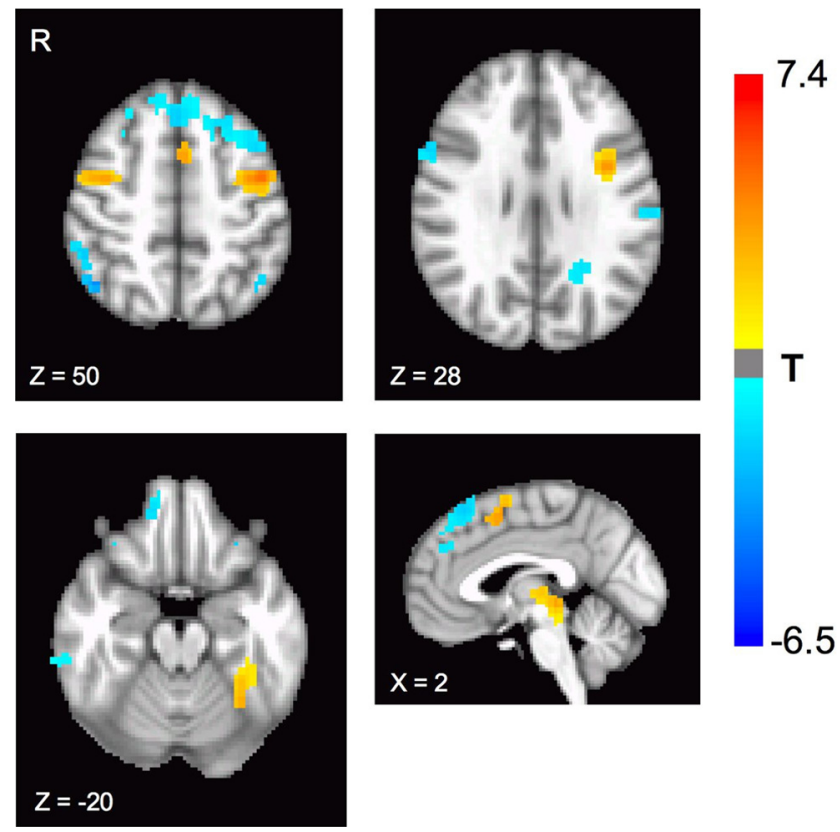

6.5

Figure 3. Main effect of task and drug. Tolcapone produced significant differences in BOLD activity during task performance relative to placebo, as assessed by the main effect of task ( $p<0.05$, corrected for multiple comparisons). Significant increases in BOLD signal could be seen in the dorsomedial prefrontal cortex and bilateral dorsolateral frontal cortices (slices $Z=50$ and $Z=28$ ), as well as the bilateral fusiform cortex (slice $Z=-20$ ) and the dorsal midbrain (sagittal slice $X=-2$ ). Areas with significant decreases in BOLD signal were also present (Table 1).

pone versus the placebo condition for Want trials $\left(-0.04 ; t_{(22)}=\right.$ $-2.4 ; p=0.025)$. As evident in Figure $2 a$, this mean reduction in ICR was also accompanied by variability in the individual responses to tolcapone. Across subjects, this variability correlated inversely with baseline impulsivity, consistent with an invertedU-shaped influence of tolcapone on delay discounting behavior (Cools and D'Esposito, 2011). Specifically, subjects with greater scores (i.e., higher impulsivity) on the BIS demonstrated larger declines in the variance-stabilized ICR $(r=-0.45 ; p=0.032$; Fig. $2 b$ ), while subjects with lower BIS scores demonstrated either smaller declines or increases in ICR. This result was not due to ceiling or floor effects in which high-ICR subjects could only demonstrate reductions in ICR and low-ICR subjects could only demonstrate increases. If these effects were present, the magnitude of the mean ICR across tolcapone and placebo conditions should be negatively correlated with the change in ICR. However, this correlation was nonsignificant $(r=-0.16 ; p=0.45)$. Moreover, these differences were not due to nonspecific dopaminergic effects on motor responding. Responses in the Don't Want condition, a test for motor impulsivity, correlated strongly with those in the Want condition $\left(r=0.98 ; p \ll 10^{-5}\right)$ across both drug conditions. Additionally, in a simple speeded response task, no difference was seen in reaction time for either visual or auditory responding on tolcapone compared with placebo (all $t_{(18)}<1.36$; $p>0.19)$. Finally, subjects themselves were unable to distinguish between tolcapone and placebo administration based on confidence ratings $\left(t_{(22)}=-0.32 ; p=0.75\right)$.

Tolcapone administration was also accompanied by neurophysiological changes. If tolcapone preferentially increases dopaminergic tone in the frontal cortex and dopamine in turn produces a net change in neural activity, active regions in the frontal cortex should show increased BOLD activity when the tolcapone condition is contrasted with the placebo condition,
Table 1. Significant regions identified by fMRI in the analyses of Figures 3,4 , and 5 (all $p<0.05$, corrected for multiple comparisons)

\begin{tabular}{|c|c|c|c|c|c|c|c|}
\hline & MNI-X & $\mathrm{MNI}-Y$ & MNI-Z & $\begin{array}{l}\text { No. } \\
\text { voxels }\end{array}$ & $t$ value & $p$ value & $B A$ \\
\hline \multicolumn{8}{|c|}{$\begin{array}{l}\text { Contrast: main effect of task, } \\
\text { tolcapone versus placebo } \\
\text { (Fig. 3) }\end{array}$} \\
\hline \multicolumn{8}{|l|}{ Area-Pos. } \\
\hline L fus gyrus & -30 & -87 & -12 & 286 & 7.24 & $6.7 \times 10^{-7}$ & 18 \\
\hline L prec gyrus & -42 & -3 & 48 & 233 & 6.51 & 0.000002 & 6 \\
\hline Midbrain & 0 & -30 & -6 & 224 & 6.62 & 0.000001 & - \\
\hline R prec gyrus & 39 & -3 & 51 & 68 & 4.74 & 0.0001 & 6 \\
\hline LSMA & -3 & 6 & 54 & 54 & 4.43 & 0.0002 & 6 \\
\hline \multicolumn{8}{|l|}{ Area-Neg. } \\
\hline B SFG & 0 & 30 & 57 & 519 & -6.46 & 0.000002 & 8 \\
\hline R MFG & 42 & 54 & -12 & 343 & -5.19 & 0.00003 & 11 \\
\hline LMOG & -21 & -81 & 3 & 143 & -5.52 & 0.00002 & 17 \\
\hline LSFG & -36 & 54 & -15 & 140 & -5.42 & 0.00002 & 11 \\
\hline LSTG & -57 & 0 & 3 & 128 & -4.50 & 0.0002 & 22 \\
\hline RIPL & 45 & -60 & 57 & 112 & -4.23 & 0.0003 & 40 \\
\hline LSPL & -27 & -45 & 72 & 92 & -4.36 & 0.0003 & 5 \\
\hline LIPL & -42 & -54 & 60 & 80 & -4.10 & 0.0005 & 40 \\
\hline LSMG & -66 & -24 & 24 & 65 & -3.94 & 0.0007 & 2 \\
\hline RITG & 63 & -33 & -18 & 38 & -5.21 & 0.00003 & 20 \\
\hline
\end{tabular}

Contrast: correlation, Want (tolcapone-placebo) with ICR (tolcapone-placebo) (Fig. 4)

Area-Neg.

Lant insula

L postc gyrus

L putamen

Contrast: Want Later-Want Now (Fig. 5a)

Area-Pos.

L ant insula

$R$ mid cing

Area-Neg.

$R$ postc gyrus

Contrast: resting state, striatal seed, tolcapone-placebo (Fig. 5b)

Area-Pos.

$\begin{array}{rrrrrlr}-42 & 18 & -6 & 64 & -4.92 & 0.00007 & 47 \\ -39 & -27 & 66 & 36 & -4.72 & 0.0001 & 3 \\ -24 & 3 & -9 & 30 & -3.55 & 0.002 & -\end{array}$
L pgACC $\begin{array}{llllll}-6 & 36 & 6 & 14 & -4.18 & 0.0004\end{array}$ 24

The $t$ statistics and associated probability values, as well as approximate Brodmann areas (BAs) estimated by the Talairach Daemon (http://www.talairach.org/daemon.html), are displayed for the peak voxel within each cluster. Abbreviations: cing, cingulate; fus, fusiform; prec, precentral; postc, postcentral; pgACC, pregenual anterior cingulate cortex; IPL, inferior parietal lobule; ITG, inferior temporal gyrus; MFG, middle frontal gyrus; MOG, middle occipital gyrus; SFG, superior frontal gyrus; SMA, supplementary motor area; SMG, supramarginal gyrus; SPL, superior parietal lobule; STG, superior temporal gyrus.

regardless of specific conditions of the delay discounting task. As shown in Figure 3 and Table 1, analysis of the main effect of task demonstrated a significant BOLD increase in specific task-active regions of the frontal cortex when tolcapone was administered. In particular, areas within the dorsolateral and medial frontal cortices were significantly more active, as were presumptive visual association areas in the bilateral fusiform gyrus, and the dorsal midbrain. Significant decreases were also seen, including in areas previously associated with the default mode network (Table 1).

To search more specifically for links between drug effects on delay discounting behavior and brain activity in individual subjects, we correlated the change in BOLD activity for Want trials (tolcapone minus placebo) with the change in ICR (tolcapone minus placebo) within a mask encompassing all frontal, cingulate, opercular, and striatal regions anterior to the primary motor cortex (see Materials and Methods). As shown in Figure 4, this 
analysis uncovered significant negative correlations in the left anterior insula, left ventral putamen, and left frontal cortex. These correlations indicate that greater decreases in ICR on tolcapone are associated with greater increases in BOLD activity in these regions, and suggest that activity in these regions contributes to the selection of a large but delayed reward. Our subsequent analyses (Fig. 5) focused in particular on the insula and the striatum, both of which have been implicated in the pathogenesis of impulse control disorders (Koob and Volkow, 2010).

Additional analyses reflected the potential importance of these regions for task performance. When BOLD activity for later choices was contrasted with BOLD activity for sooner choices across the group, regardless of drug condition, greater activity was seen in an overlapping region in the left anterior insula, as well as the dorsal anterior cingulate (Fig. 5a). To evaluate the contribution of frontostriatal connectivity changes related to tolcapone and unconfounded by task performance, we calculated the resting state connectivity of the left ventral putamen with the prefrontal cortex (see Materials and Methods). This analysis demonstrated a significantly decreased correlation between the left ventral putamen and the pregenual cingulate in the tolcapone versus placebo condition (Fig. 5b). Importantly, when we subsequently evaluated the strength of the coherence between these regions in the task state, the coherence between the putamen and the pregenual cingulate varied significantly with the change in ICR $(r=0.64 ; p=0.0017$; Fig. $5 c)$. In other words, tolcapone treatment reduced resting state connectivity between the left putamen and the pregenual cingulate; and during task performance, tolcapone-induced reductions in the strength of the connectivity between these two regions correlated with reduced impulsivity.

\section{Discussion}

Human genetic data indicate that greater COMT activity increases impulsivity (Boettiger et al., 2007; Paloyelis et al., 2010). Because COMT significantly degrades cortical but not striatal dopamine, this genetic finding suggests that lower cortical dopamine tone promotes impulsive behaviors. Our present finding that COMT inhibition decreases preference for smaller, sooner rewards directly supports this hypothesis. Consistent with this idea and data that delay discounting and trait impulsivity load onto the same psychological factor (Meda et al., 2009), larger tolcapone-induced decreases in impulsive choice were found in subjects with greater baseline impulsivity, as measured by the Barratt Impulsivity Scale (Patton et al., 1995). This finding is also consistent with previous reports that the effects of other dopaminergic agents, such as the $\mathrm{D}_{2}$ receptor agonist bromocriptine, depend on baseline impulsivity levels. In a working memory paradigm, Cools et al. (2007) demonstrated that bromocriptine improved the updating of working memory in high-impulsive but not low-impulsive subjects. In keeping with that result, we find that tolcapone reduced impulsivity more in high-impulsive subjects. It is possible that these findings reflect the existence of an inverted-U-shaped curve governing dopamine effects (Cools and D'Esposito, 2011), in which optimal dopaminergic tone can either be reached or "overdosed" by a drug that increases dopamine tone, depending on subjects' baseline dopamine levels. Consistent with this hypothesis, a behavioral study of prepulse inhibition demonstrated that subjects with a more active form of the COMT enzyme (and thus lower cortical dopamine tone) showed improved performance on tolcapone, but those with a less active form did not (Giakoumaki et al., 2008). Similarly, in a study of executive function, Apud et al. (2007) found that subjects with a more active form of COMT showed greater improvements on tests of set shifting and verbal episodic memory when administered tolcapone. These results are in keeping with our finding that subjects with higher baseline impulsivity may benefit the most from tolcapone.

Our data also provide direct evidence in humans that COMT regulation of dopamine levels in the prefrontal cortex is functionally significant. Tolcapone treatment increased BOLD signal in the dorsolateral and dorsomedial frontal cortices and decreased signal in other cortical areas associated with the default mode network. Importantly, the current functional imaging findings suggest a circuit mechanism for dopamine effects on intertemporal choice. Correlating tolcapone-associated decreases in impulsive choice ratio with changes in BOLD activity revealed significant changes in two regions of the left hemisphere-the striatum and the anterior insula-that have been implicated in the pathogenesis of substance abuse (Naqvi et al., 2007; Goldstein et al., 2009; Koob and Volkow, 2010), a prevalent form of impulse control disorder. Furthermore, the tolcapone-induced changes in the left ventral putamen were associated with a decrease in connectivity between the putamen and the pregenual anterior cingulate that varied directly with a decrease in ICR.

Consistent with its central role in maintenance of task set across time (Dosenbach et al., 2006), the anterior insula showed greater activity when later rewards were selected. Intriguingly, this same region demonstrated relative hypoactivity in marijuana-abusing subjects with failures of error 
a
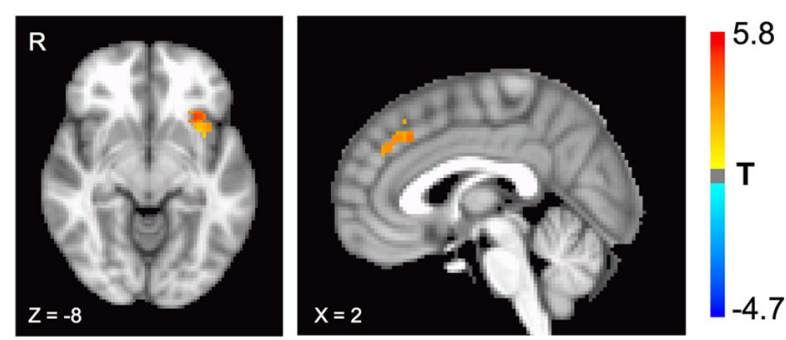

b

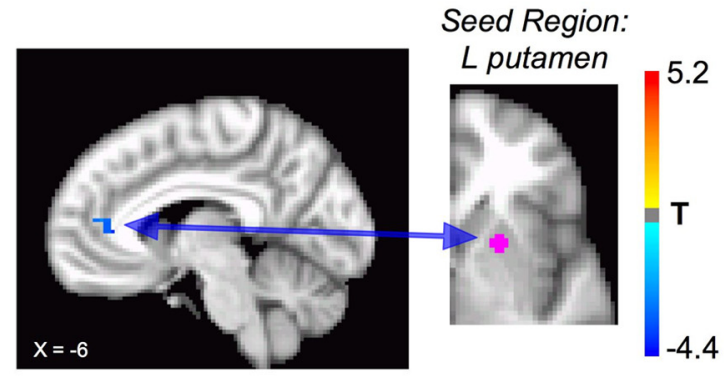

C

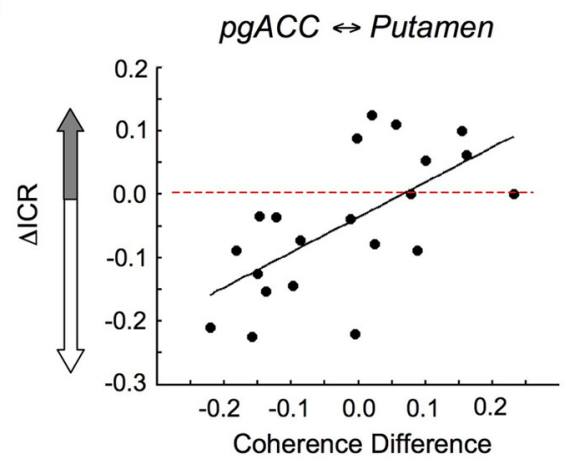

Figure 5. Insula and putamen function/connectivity. $\boldsymbol{a}$, Shown is the contrast between all Want (Later) and all Want (Now) choices ( $p<0.05$, corrected for multiple comparisons). This contrast revealed a significant increase in BOLD signal in the left anterior insula, overlapping the anterior insula region identified in Figure 4 (Table 1). $\boldsymbol{b}$, In the resting state, the correlation between the putamen region identified in Figure 4 and the pregenual anterior cingulate cortex ( $p g A C C$ ) declined ( $p<0.05$, corrected for multiple comparisons; sagittal slice $X=4)$ ). c, During task performance, the difference in coherence between the $\mathrm{pgACC}$ and left putamen on tolcapone versus placebo correlated positively with the change in ICR $(r=0.64 ; p=0.0017)$. Specifically, greater declines in $\mathrm{pgACC} \leftrightarrows$ putamen coherence correlated with greater declines in ICR.

awareness (Hester et al., 2009). These results are interesting because lesions in the insula, which would be expected to reduce function, can disrupt nicotine addiction in humans (Naqvi et al., 2007) and amphetamine seeking in rats (Contreras et al., 2007). The similarity of the effects associated with increased activity and lesion could be explained by anatomical differences in insular function (i.e., in both of the latter cases, lesions may have preferentially involved the posterior insula, whereas the activations in our study were located in the anterior insula). Given the heterogeneity of insular function (Craig, 2009; Nieuwenhuys, 2012), it is possible that this anatomical difference may partially explain why greater BOLD activity in the anterior insula was associated with decreased discounting of later rewards - a reduction that would be hypothesized to ameliorate addictive behaviors - rather than an increase in discounting, as might be predicted from the lesion data. Alternatively, increases in BOLD signal reflect increases in metabolic activity, not necessarily excitatory activity, and it is also conceivable that such ac- tivity may relate to greater activity of inhibitory circuits within this region.

Perhaps more surprising was our finding that increases in activity in the ventral putamen were associated with declines in impulsive choice. While the more ventral striatum is typically linked to reward processing, relatively more dorsal regions are linked to cortical circuits that engage the dorsal anterior cingulate and dorsolateral frontal cortices (Haber and Knutson, 2010). In the tolcapone condition, this region in the left ventral putamen showed less connectivity with the pregenual cingulate, consistent with weaker striatal connectivity when dopamine levels are raised in "valuation-related" cortical regions. Given that the pregenual cingulate has been previously associated with assessments of value (Peters and Büchel, 2010b) and impulsivity (Hinvest et al., 2011), the extent to which connectivity with the pregenual ACC declined correlated with decline in ICR, arguing that a relative shift in connectivity of this striatal subregion may indeed favor the selection of longer-term rewards. Moreover, it is important to note that even anatomically overlapping BOLD activations in striatum might conceivably be associated with different cognitive effects. In the striatum, rodent studies have demonstrated two main populations of neurons with overlapping anatomical distributions: those that primarily express either dopamine $\mathrm{D}_{1}$-like or dopamine $\mathrm{D}_{2}$-like type receptors. Activity in these two populations has opposing effects on behaviors; $\mathrm{D}_{1}$ receptor-expressing neurons promote, while $\mathrm{D}_{2}$ receptor-expressing neurons inhibit reward-seeking behaviors (Maia and Frank, 2011).

Importantly, the above behavioral and neural results are likely to reflect the relative selectivity of tolcapone, both pharmacologically and neuroanatomically. Animal studies argue that (1) unlike drugs that alter the synaptic function of multiple catecholamines, tolcapone likely produces relatively specific increases in dopamine; and (2) because of the importance of COMT for cortical dopamine metabolism, tolcapone is likely to have its greatest effect in anterior cortical regions including the frontal cortex and insula. With respect to the first point, deletion of the COMT gene in mice preferentially increased levels of dopamine, as opposed to norepinephrine or other catecholamines (Gogos et al., 1998). In rats, tolcapone itself potentiated increases in dopamine release within prefrontal cortex without changing the release of norepinephrine (Tunbridge et al., 2004). However, amphetamine increases extracellular dopamine as well as serotonin and norepinephrine (Kuczenski and Segal, 1997), and L-dopa can alter extracellular norepinephrine levels in frontal cortex (Dayan and Finberg, 2003). With respect to the underlying neuroanatomy, tolcapone-induced increases in dopamine tone in mice were regionally specific (i.e., they were observed in frontal cortex but not striatum) (Gogos et al., 1998). In contrast, PET studies of both amphetamine (Narendran et al., 2010) and L-dopa (Vernaleken et al., 2007) in humans demonstrate significant changes in dopamine metabolism in the striatum. These findings suggest that COMT inhibition via tolcapone may represent a relatively specific way to increase dopamine, but not other catecholamine, tone in frontal cortex but not subcortical regions.

Nonpharmacological interventions can also decrease impulsivity. Engaging episodic memory by asking subjects to associate a specific event with future rewards increases selection of those later rewards (Peters and Büchel, 2010a), and working memory training in stimulant-addicted patients also leads to a reduction in delay discounting behavior (Bickel et al., 2011). Our imaging findings can potentially extend our understanding of the neural basis of these results, in that tolcapone led to greater activity in regions, including the anterior insula, that have been hypothe- 
sized to reflect more deliberative cognitive processes. These results also raise the possibility that tolcapone could be usefully combined with such nonpharmacological interventions to enhance behavioral therapies. A case report is supportive of these arguments: after failing multiple previous medications, a patient with a history of compulsive shoplifting successfully reduced her urge to steal when tolcapone was combined with behavioral therapy (Grant, 2011).

In conclusion, tolcapone reduces impulsivity, particularly in high-impulsive individuals, through its ability to change activity in, or connectivity with, insular and frontal brain regions so as to promote more deliberative decision making (Bechara, 2005). An important step for future studies would be to assess whether COMT genotype influences the effects of tolcapone: specifically, whether subjects with a more active COMT enzyme, as predicted by the Val158Val genotype, show greater declines in impulsive choice on tolcapone. Additionally, for treatment of addictive behaviors - whether for substances, gambling, or other rewards - it is essential to determine whether declines in impulsive choice on a delay discounting task correlate with real-world improvements in substance use and other impulse control disorders. For example, naltrexone, a nonselective opioid antagonist that decreases impulsive responding in a delay discounting task (Boettiger et al., 2009), is also FDA-approved for the treatment of alcoholism (O’Malley et al., 1992; Volpicelli et al., 1992). If tolcapone reduces "real-world" impulsive behaviors, these data indicate that it could be an effective adjunct to current behavioral and pharmacological therapies, and thereby provide an additional treatment option for these pervasive and costly illnesses.

\section{References}

Apud JA, Mattay V, Chen J, Kolachana BS, Callicott JH, Rasetti R, Alce G, Iudicello JE, Akbar N, Egan MF, Goldberg TE, Weinberger DR (2007) Tolcapone improves cognition and cortical information processing in normal human subjects. Neuropsychopharmacology 32:1011-1020.

Bechara A (2005) Decision making, impulse control and loss of willpower to resist drugs: a neurocognitive perspective. Nat Neurosci 8:1458-1463.

Bickel WK, Yi R, Landes RD, Hill PF, Baxter C (2011) Remember the future: working memory training decreases delay discounting among stimulant addicts. Biol Psychiatry 69:260-265.

Boettiger CA, Mitchell JM, Tavares VC, Robertson M, Joslyn G, D'Esposito M, Fields HL (2007) Immediate reward bias in humans: fronto-parietal networks and a role for the catechol-O-methyltransferase $158(\mathrm{Val} / \mathrm{Val})$ genotype. J Neurosci 27:14383-14391.

Boettiger CA, Kelley EA, Mitchell JM, D’Esposito M, Fields HL (2009) Now or Later? An fMRI study of the effects of endogenous opioid blockade on a decision-making network. Pharmacol Biochem Behav 93:291-299.

Carter RM, Meyer JR, Huettel SA (2010) Functional neuroimaging of intertemporal choice models: a review. J Neurosci Psychol Econ 3:27-45.

Chen J, Lipska BK, Halim N, Ma QD, Matsumoto M, Melhem S, Kolachana BS, Hyde TM, Herman MM, Apud J, Egan MF, Kleinman JE, Weinberger DR (2004) Functional analysis of genetic variation in catechol- $O$ methyltransferase (COMT): effects on mRNA, protein, and enzyme activity in postmortem human brain. Am J Hum Genet 75:807-821.

Coffey SF, Gudleski GD, Saladin ME, Brady KT (2003) Impulsivity and rapid discounting of delayed hypothetical rewards in cocaine-dependent individuals. Exp Clin Psychopharmacol 11:18-25.

Contreras M, Ceric F, Torrealba F (2007) Inactivation of the interoceptive insula disrupts drug craving and malaise induced by lithium. Science 318:655-658.

Cools R (2008) Role of dopamine in the motivational and cognitive control of behavior. Neuroscientist 14:381-395.

Cools R, D'Esposito M (2011) Inverted-U-shaped dopamine actions on human working memory and cognitive control. Biol Psychiatry 69:e113-e125.

Cools R, Sheridan M, Jacobs E, D’Esposito M (2007) Impulsive personality predicts dopamine-dependent changes in frontostriatal activity during component processes of working memory. J Neurosci 27:5506-5514.
Craig AD (2009) How do you feel—now? The anterior insula and human awareness. Nat Rev Neurosci 10:59-70.

Dagher A, Robbins TW (2009) Personality, addiction, dopamine: insights from Parkinson's disease. Neuron 61:502-510.

Dayan L, Finberg JP (2003) L-DOPA increases noradrenaline turnover in central and peripheral nervous systems. Neuropharmacology 45: $524-533$.

de Wit H, Enggasser JL, Richards JB (2002) Acute administration of D-amphetamine decreases impulsivity in healthy volunteers. Neuropsychopharmacology 27:813-825.

Dosenbach NU, Visscher KM, Palmer ED, Miezin FM, Wenger KK, Kang HC, Burgund ED, Grimes AL, Schlaggar BL, Petersen SE (2006) A core system for the implementation of task sets. Neuron 50:799-812.

Fox MD, Snyder AZ, Vincent JL, Corbetta M, Van Essen DC, Raichle ME (2005) The human brain is intrinsically organized into dynamic, anticorrelated functional networks. Proc Natl Acad Sci U S A 102:9673-9678.

Giakoumaki SG, Roussos P, Bitsios P (2008) Improvement of prepulse inhibition and executive function by the COMT inhibitor tolcapone depends on COMT Val158Met polymorphism. Neuropsychopharmacology 33:3058-3068.

Gogos JA, Morgan M, Luine V, Santha M, Ogawa S, Pfaff D, Karayiorgou M (1998) Catechol-O-methyltransferase-deficient mice exhibit sexually dimorphic changes in catecholamine levels and behavior. Proc Natl Acad Sci U S A 95:9991-9996.

Goldstein RZ, Craig AD, Bechara A, Garavan H, Childress AR, Paulus MP, Volkow ND (2009) The neurocircuitry of impaired insight in drug addiction. Trends Cogn Sci 13:372-380.

Grant JE (2011) Kleptomania treated with tolcapone, a catechol-O-methyltransferase (COMT) inhibitor. Prog Neuropsychopharmacol Biol Psychiatry 35:295-296.

Haber SN, Knutson B (2010) The reward circuit: linking primate anatomy and human imaging. Neuropsychopharmacology 35:4-26.

Hester R, Nestor L, Garavan H (2009) Impaired error awareness and anterior cingulate cortex hypoactivity in chronic cannabis users. Neuropsychopharmacology 34:2450-2458.

Hinvest NS, Elliott R, McKie S, Anderson IM (2011) Neural correlates of choice behavior related to impulsivity and venturesomeness. Neuropsychologia 49:2311-2320.

Kang MJ, Rangel A, Camus M, Camerer CF (2011) Hypothetical and real choice differentially activate common valuation areas. J Neurosci 31:461-468.

Kayser AS, Sun FT, D'Esposito M (2009) A comparison of Granger causality and coherency in fMRI-based analysis of the motor system. Hum Brain Mapp 30:3475-3494.

Kirby KN, Petry NM, Bickel WK (1999) Heroin addicts have higher discount rates for delayed rewards than non-drug-using controls. J Exp Psychol Gen 128:78-87.

Koob GF, Volkow ND (2010) Neurocircuitry of addiction. Neuropsychopharmacology 35:217-238.

Kuczenski R, Segal DS (1997) Effects of methylphenidate on extracellular dopamine, serotonin, and norepinephrine: comparison with amphetamine. J Neurochem 68:2032-2037.

Maia TV, Frank MJ (2011) From reinforcement learning models to psychiatric and neurological disorders. Nat Neurosci 14:154-162.

Meda SA, Stevens MC, Potenza MN, Pittman B, Gueorguieva R, Andrews MM, Thomas AD, Muska C, Hylton JL, Pearlson GD (2009) Investigating the behavioral and self-report constructs of impulsivity domains using principal component analysis. Behav Pharmacol 20:390-399.

Mitchell JM, Fields HL, D’Esposito M, Boettiger CA (2005) Impulsive responding in alcoholics. Alcohol Clin Exp Res 29:2158-2169.

Naqvi NH, Rudrauf D, Damasio H, Bechara A (2007) Damage to the insula disrupts addiction to cigarette smoking. Science 315:531-534.

Narendran R, Mason NS, Laymon CM, Lopresti BJ, Velasquez ND, MayMA, Kendro S, Martinez D, Mathis CA, Frankle WG (2010) A comparative evaluation of the dopamine $\mathrm{D}_{2 / 3}$ agonist radiotracer $\left[{ }^{11} \mathrm{C}\right](-)-\mathrm{N}$-propyl-norapomorphine and antagonist $\left[{ }^{11} \mathrm{C}\right]$ raclopride to measure amphetamine-induced dopamine release in the human striatum. J Pharmacol Exp Ther 333:533-539.

Nieuwenhuys R (2012) The insular cortex: a review. Prog Brain Res 195:123-163.

O'Malley SS, Jaffe AJ, Chang G, Schottenfeld RS, Meyer RE, Rounsaville B (1992) Naltrexone and coping skills therapy for alcohol dependence. A controlled study. Arch Gen Psychiatry 49:881-887. 
Paloyelis Y, Asherson P, Mehta MA, Faraone SV, Kuntsi J (2010) DAT1 and COMT effects on delay discounting and trait impulsivity in male adolescents with attention deficit/hyperactivity disorder and healthy controls. Neuropsychopharmacology 35:2414-2426.

Patton JH, Stanford MS, Barratt ES (1995) Factor structure of the Barratt impulsiveness scale. J Clin Psychol 51:768-774.

Peters J, Büchel C (2010a) Episodic future thinking reduces reward delay discounting through an enhancement of prefrontal-mediotemporal interactions. Neuron 66:138-148.

Peters J, Büchel C (2010b) Neural representations of subjective reward value. Behav Brain Res 213:135-141.

Peters J, Büchel C (2011) The neural mechanisms of inter-temporal decision-making: understanding variability. Trends Cogn Sci 15:227-239.

Petry NM (2001) Delay discounting of money and alcohol in actively using alcoholics, currently abstinent alcoholics, and controls. Psychopharmacology (Berl) 154:243-250.

Pine A, Shiner T, Seymour B, Dolan RJ (2010) Dopamine, time, and impulsivity in humans. J Neurosci 30:8888-8896.

Rosenberg JR, Amjad AM, Breeze P, Brillinger DR, Halliday DM (1989) The Fourier approach to the identification of functional coupling between neuronal spike trains. Prog Biophys Mol Biol 53:1-31.

Rosner B (1983) Percentage points for a generalized ESD many outlier procedure. Technometrics 25:165-172.
Sellitto M, Ciaramelli E, di Pellegrino G (2010) Myopic discounting of future rewards after medial orbitofrontal damage in humans. J Neurosci 30:16429-16436.

Tunbridge EM, Bannerman DM, Sharp T, Harrison PJ (2004) Catechol-Omethyltransferase inhibition improves set-shifting performance and elevates stimulated dopamine release in the rat prefrontal cortex. J Neurosci 24:5331-5335.

Tzourio-Mazoyer N, Landeau B, Papathanassiou D, Crivello F, Etard O, Delcroix N, Mazoyer B, Joliot M (2002) Automated anatomical labeling of activations in SPM using a macroscopic anatomical parcellation of the MNI MRI single-subject brain. Neuroimage 15:273-289.

Vernaleken I, Buchholz HG, Kumakura Y, Siessmeier T, Stoeter P, Bartenstein P, Cumming P, Gründer G (2007) "Prefrontal" cognitive performance of healthy subjects positively correlates with cerebral FDOPA influx: an exploratory $\left[{ }^{18} \mathrm{~F}\right]$-fluoro-L-DOPA-PET investigation. Hum Brain Mapp 28:931-939.

Volkow ND, Fowler JS, Wang GJ, Baler R, Telang F (2009) Imaging dopamine's role in drug abuse and addiction. Neuropharmacology 56 [Suppl 1]:3-8.

Volpicelli JR, Alterman AI, Hayashida M, O’Brien CP (1992) Naltrexone in the treatment of alcohol dependence. Arch Gen Psychiatry 49:876880 . 